\title{
ScienceDirect
}

\section{Robust control of a system with a pneumatic spring}

\author{
Dongwon Kim ${ }^{\mathrm{a}, \mathrm{b}, *}$, Jinoh $\mathrm{Lee}^{\mathrm{c}}$ \\ ${ }^{a}$ Department of Physical Therapy and Rehabilitation Science, School of Medicine, University of Maryland, \\ Baltimore, Maryland 21201, United States \\ ${ }^{\mathrm{b}}$ Department of Biongineering, School of Engineering, University of Maryland, College Park, Maryland 20742, \\ United States \\ ${ }^{\mathrm{c}}$ Institute of Robotics and Mechatronics, German Aerospace Center (DLR), Wessling, Bavaria 82234, Germany
}

Received 2 December 2019; received in revised form 19 August 2020; accepted 27 October 2020

Available online xxx

\begin{abstract}
Recently, series elasticity has been realized using pneumatics in human-robot interaction systems. Pneumatic circuits provide not only a flexible power transmission, but also the elastic element in a series elastic actuator (SEA). Pneumatic series elastic systems involve more than twice the number of parameters that influence system behaviors in comparison with rigid robotic systems. In this study, a position controller that eliminates the need of identifying a system model by employing the time delay estimation (TDE) technique is proposed for pneumatic SEA systems. The TDE technique is effective in compensating for system dynamics and all uncertainties involved in system behaviors without imposing computation load. TDE error is cancelled out through a learning way, which improves control performance and leads to asymptotic stability. A simulation study demonstrates the robustness of the proposed controllers against uncertainties imposed on the motor system as well as uncertainties on the end-effector. The simulation shows the efficacy of the learning compensation for TDE error.

(C) 2020 The Franklin Institute. Published by Elsevier Ltd. All rights reserved.
\end{abstract}

\section{Introduction}

Series elastic actuators (SEAs) are used in robot designs because of its safety feature to humans interacting with robots [1-7]. In SEAs, compliance is purposefully introduced

\footnotetext{
* Corresponding author at: Department of Physical Therapy and Rehabilitation Science, School of Medicine, University of Maryland, Baltimore, MD 21201, United States.

E-mail address: dongwonkim@som.umaryland.edu (D. Kim).
} 
between an actuator and robot end-effector and masks the high impedance of the actuator from humans or environments. SEAs are able to maintain low impedance at high frequencies, while they offer an ability to shape the programmed impedance within their operational bandwidth. Recently, it has been noticed that actuation that employs compressible fluid takes over the advantages of SEAs in that fluid compressibility provides elasticity between a bulky and heavy actuator and end-effector [8-10]. A pneumatic circuit hides the actuator's impedance from the end-effector, reducing the backdrive impedance of the device. Impact forces on the end-effector are absorbed by fluid compressibility. As well, pneumatic actuation provides flexible transmission and high force-to-weight ratios.

SEAs are a non-collocated system since the sensor and actuator used for control are separated by the elastic structure [11]. The position of the end-effector is controlled by the deformation of the elastic structure and the deformation is controlled by the actuator. These high-order systems consist of two masses (one is the actuator and the other is the end-effector) but they are controlled typically with only one control command. As each mass involves friction and disturbance, the number of parameters that characterise an SEA system doubles in comparison with a rigid actuation system. Moreover, the property of the elastic element determines the behaviors of the SEA system. For satisfactory control of an SEA system, these parameters might require to be well identified. Even in use of proportional-integral-derivative (PID) type controllers [12-14], the property of the elastic element needs to be known; it is a necessary procedure to control the deformation of the elastic structure.

Like rigid robot manipulators, various position control solutions for SEA systems (or flexible-joint systems) have been proposed to suppress the influence of uncertainties over system modelling on control performance. Most of these solutions are realized by using sliding mode techniques, intelligent algorithms (fuzzy logic and neural networks) or their combinations [15-22]. However these approaches require at least the nominal value of each parameter of the target system. In some controllers, more complicated schemes are employed to deal with unstructured uncertainties, which would lead to substantial computation load.

Time delay estimation (TDE) has been recognized as a powerful tool in the control field [23-34]. The TDE technique uses the command (input) and its accompanying system state (output) to quantitatively estimate system dynamics and disturbances that are difficult to identify precisely. The estimate that is obtained using the previous-step sensor reading and record of previous-step command can be used to cancel out system dynamics and uncertainties through their incorporation into the control at the current step. Consequently, employing the TDE technique leads to accuracy and robustness of control in the presence of a wide class of uncertainties including both structured and unstructured uncertainties under the assumption of infinitesimally small sampling intervals. Also TDE alleviates computation load for the system dynamics and uncertainties in comparison with other robust control schemes. Note that the term time delay estimation used in this paper has a different meaning from one used in several papers where the term indicates estimation of an uncertain time delay in feedback loops [35].

In this study, a TDE-incorporated position controller is proposed for a pneumatic SEA system. This controller does not require a precise knowledge of the target system. In particular, the TDE technique eliminates the need to identify elasticity placed between an actuator and robot link that is highly nonlinear and even time-varying in a pneumatic SEA system. Without significant computation load, the proposed controller exhibits robustness to mismatched uncertainties (e.g., friction and disturbance imposed on the end-effector) as well as matched uncertainties (e.g., friction and disturbance imposed on the actuator). 


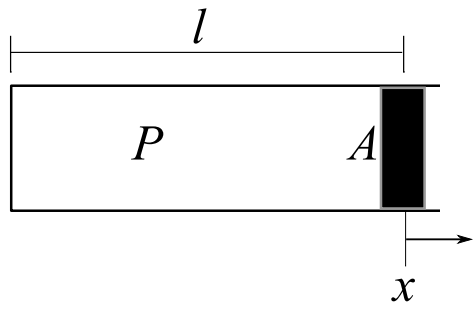

(a)

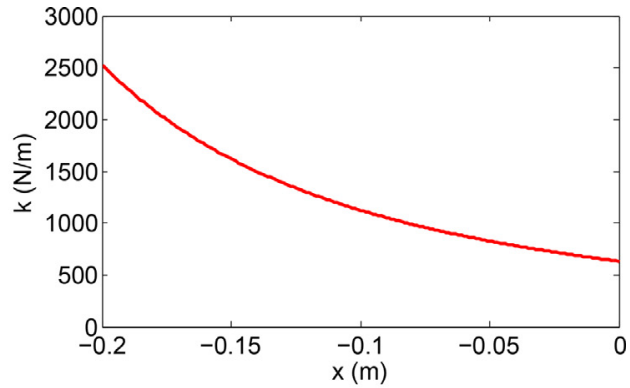

(b)

Fig. 1. (a) Schematic of a cylinder and piston and (b) the curve of stiffness of air of $0.001 \mathrm{~m}^{3}$ with piston area $A=0.0025 \mathrm{~m}^{2}$ at $20{ }^{\circ} \mathrm{C}(T=293 \mathrm{~K})$. The parameters are selected as $l=0.4 \mathrm{~m}, m=0.0012 \mathrm{~kg}$, and $R=287 \mathrm{~J} / \mathrm{kgK}$.

In addition to the TDE technique, a learning algorithm is adopted in the proposed controller to cope with time delay estimation error (TDE error), which can be regarded as the biggest challenge to be resolved in controllers with TDE. TDE error, which arises from approximating the current system dynamics and uncertainties with the values of control input and state at the previous-time step, exerts a critical influence on both stability and control performance [26,27]. Typically, TDE-incorporated controllers achieve uniformly ultimate boundedness at the best case, with the assumption that TDE error is bounded. The learning algorithm compensates for TDE error by estimating it. Employing the learning counteraction for TDE error opens the possibility that the system is asymptotically stabilized beyond uniformly ultimate bounded stability.

In this study, the controller is developed in a sampled-data environment. Controllers incorporated with the TDE technique have been designed in the continuous-time framework, although these controllers need to be run using digital computers to control continuous-time systems [24-31,36,37]. The design procedures and stability analyses of almost all controllers with TDE overlooked the sampled-data environment. These controllers were developed forming TDE with a control command and its corresponding system state which are at the same time point. We assume that the command and state at the same time point are in causality relationship in the continuous-time framework, but they are not in practice. An input and an output at the same time point do not reflect system dynamics. Therefore, the need to design and analyze a TDE-incorporated controller for sampled-data systems, with practical causality about the control command and its output, arises.

\section{Target system: SEA with a pneumatic circuit}

It is well known that air is typically compressible [38]. A container filled with air changes volume when it is pressed, while the container tends to return to its original state if the applied force is removed. The air in the container acts as a spring. Fig. 1(a) depicts a cylinder filled with $0.001 \mathrm{~m}^{3}$ of air. The force $F$ applied to the piston by the air is the product of the pressure inside the chamber $P$ and the area of the piston $A$, given as

$F=P A$. 
(a)
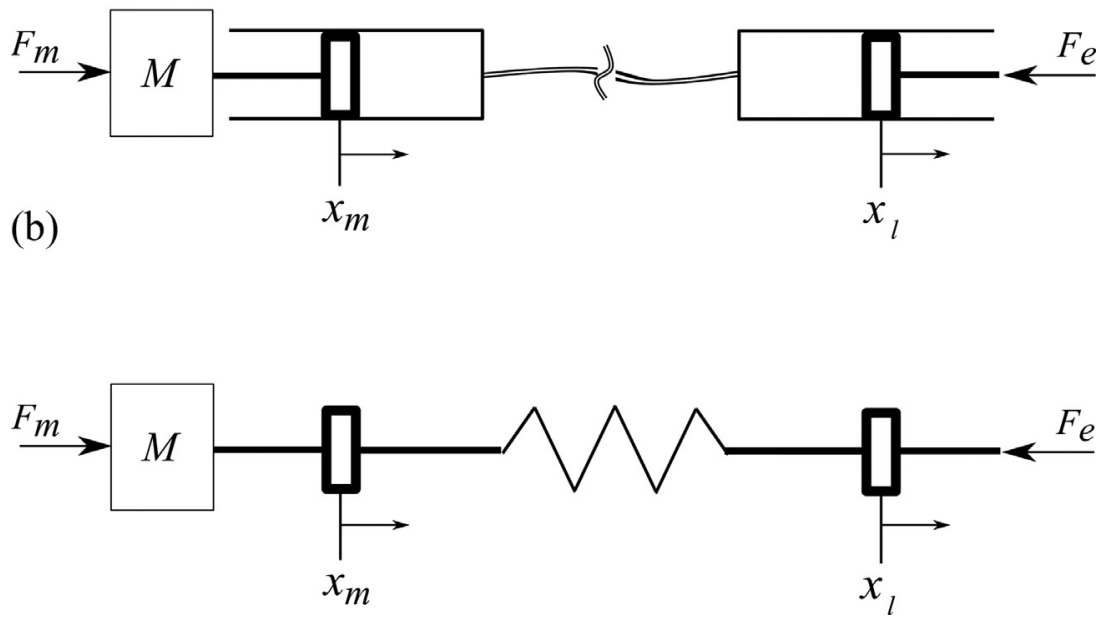

Fig. 2. (a) Actuation with pneumatic circuits, and (b) series elastic actuation.

The stiffness effect of the fluid in the chamber can be obtained by differentiating the force $F$ with respect to the position $x$ as follows:

$k \triangleq-\frac{\partial F}{\partial x}=-\frac{\partial P}{\partial x} A$,

with the following relationship coming from the assumption that the air in the cylinder is an ideal gas undergoing an isothermal process $(\Delta T=0)$,

$P=\frac{m R T}{V}=\frac{m R T}{A(l+x)}$,

where $m, V$ are the mass and volume of the entrapped air, respectively; $R$ the universal gas constant; $T$ the absolute temperature and $l$ the length of the cylinder. The partial derivative of $P$ with respect to $x$ is then obtained as

$\frac{\partial P}{\partial x}=-\frac{m R T}{A(l+x)^{2}}$.

Substituting Eq. (4) into Eq. (2) yields

$k=\frac{m R T}{(l+x)^{2}}$.

The stiffness $k$ thus increases nonlinearly as the piston is pushed in, as shown in Fig. 1(b).

Including a container filled with compressible air between the actuator and end-effector depicted in Fig. 2(a) provides the primary features of a series elastic actuator (SEA) shown in Fig. 2(b), as experimentally investigated in [8]. A motor input $F_{m}$ produces the displacement of mass $M$, defined as $x_{m}$, and accordingly results in the deformation of the elastic structure. The deformation brings about the displacement of the end-effector, defined as $x_{l}$. The end-effector interacts with the environment through a force $F_{e}$.

In this study, the target system to be controlled is an arm exoskeleton with pneumatic circuits, as depicted in Fig. 3. The master cylinder is powered by a motor, as in [8], and 


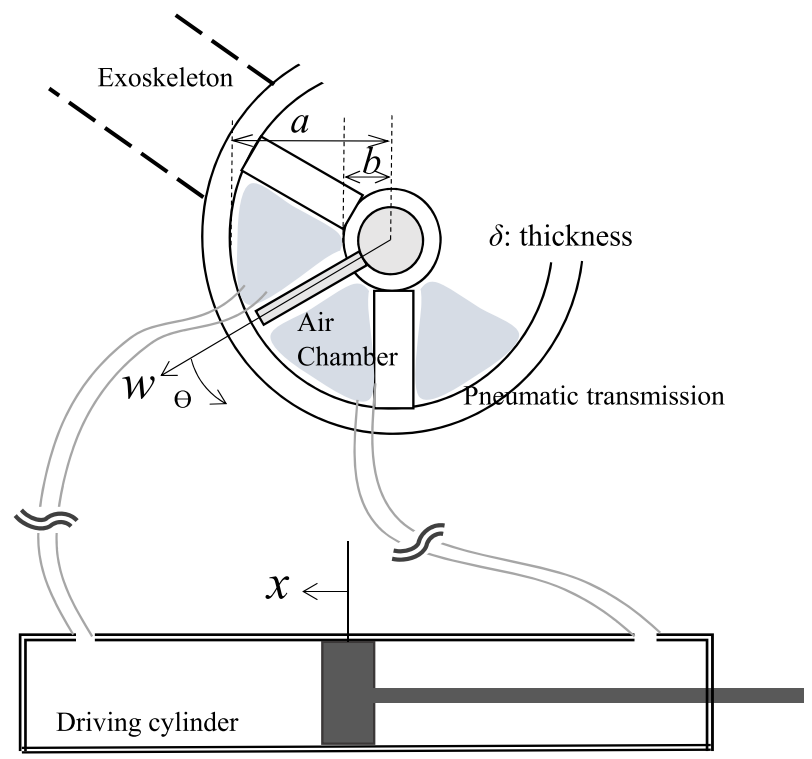

Fig. 3. (a) Schematic of an arm exoskeleton with pneumatic circuits.

the end-effector is actuated through pneumatic circuits connected to the rotary transmission designed in [9].

With the translational and rotational stiffnesses resulting from pneumatic compressibility lumped as $k_{x}$ and $k_{\theta}$, respectively, the target system can be modeled based on SEA systems or flexible-joint systems [39] as follows:

$$
\begin{gathered}
I \ddot{\theta}=k_{\theta}(x / r-\theta)-m g h \sin \theta+F_{f 1}+D_{1}, \\
B \ddot{x}+k_{x}(x-r \theta)=F_{f 2}+D_{2}+F_{m},
\end{gathered}
$$

where $I$ and $m$ denote the inertias of the driven part including the exoskeleton, respectively; $g$ the gravitational constant; $h$ the distance between the joint and the center of mass of the driven part; $B$ the inertia of the driving part; $r$ the ratio between translational motion and rotational motion. The control input $F_{m}$ is exerted on the driving part. $F_{f 1}$ and $F_{f 2}$ denote friction forces that consist of viscous friction and Coulomb friction, respectively. $D_{1}$ and $D_{2}$ are bounded oscillatory disturbances that result from sensor noise and communication noise, respectively.

The stiffnesses $k_{x}$ and $k_{\theta}$ can be obtained using the ideal gas law $P V=m R T$. To express the stiffness $k_{x}, F_{p}$ denotes the resultant force exerted on the driving piston. The pneumatic system consists of two separate fluidic circuits: one entraps air in front of the driving piston, the other one in back of the driving piston. The first circuit is expressed with subscript 1 and the second circuit with subscript 2. It is assumed that the air chambers are made of an inelastic material.

$F_{p}=-P_{1} A_{1}+P_{2} A_{2}=-\frac{m_{1} R T}{V_{1}} A_{1}+\frac{m_{2} R T}{V_{2}} A_{2}$,

where $P, V$ and $m$ with subscript 1 and 2 denote the pressure, volume and mass of the air entrapped in each circuit respectively, $A_{1}$ is the front side area of the piston and $A_{2}$ is the back side area of the piston considering the area of the rod. 
The volumes of the air entrapped in each circuit $V_{1}, V_{2}$ are obtained as

$V_{1}=V_{10}-A_{1} x+\frac{3 \delta\left(a^{2}-b^{2}\right) \theta}{2}$,

$V_{2}=V_{20}+A_{2} x-\frac{3 \delta\left(a^{2}-b^{2}\right) \theta}{2}$,

where $V$ with subscript 0 indicates the initial volume, $\delta, a$ and $b$ denote the dimensions of the air chambers in the rotary pneumatic transmission, respectively (see Fig. 3), and weight 3 is multiplied because three air chambers form one fluidic circuit.

Then the stiffness $k_{x}$ is given as

$$
\begin{aligned}
k_{x} \triangleq & -\frac{\partial F_{p}}{\partial x} \\
& =-\frac{m_{1} R T}{V_{1}^{2}} V_{1}^{\prime} A_{1}+\frac{m_{2} R T}{V_{2}^{2}} V_{2}^{\prime} A_{2}=\frac{m_{1} R T}{V_{1}^{2}} A_{1}^{2}+\frac{m_{2} R T}{V_{2}^{2}} A_{2}^{2} \\
& =\frac{m_{1} R T}{\left(V_{10}-A_{1} x+\frac{3 \delta\left(a^{2}-b^{2}\right) \theta}{2}\right)^{2}} A_{1}^{2}+\frac{m_{2} R T}{\left(V_{20}+A_{2} x-\frac{3 \delta\left(a^{2}-b^{2}\right) \theta}{2}\right)^{2}} A_{2}^{2} .
\end{aligned}
$$

Next, the stiffness $k_{\theta}$ can be obtained in a similar way.

The torque $\tau_{t}$ that is transferred to the exoskeleton in the transmission by the fluid can be expressed as the product of the force by the air pressure and the distance of it from the the origin along the $w$ axis:

$\tau_{t}=3 P_{1} \delta \int_{b}^{a} w d w+3 P_{2} \delta \int_{b}^{a} w d w$.

Then the stiffness $k_{\theta}$ is written as

$k_{\theta} \triangleq \frac{\partial \tau_{t}}{\partial \theta}=\frac{3 \delta\left(a^{2}-b^{2}\right)}{2}\left(\frac{\partial P_{1}}{\partial \theta}-\frac{\partial P_{2}}{\partial \theta}\right)=\left(\frac{3 \delta\left(a^{2}-b^{2}\right)}{2}\right)^{2}\left(\frac{m_{1} R T}{V_{1}^{2}}+\frac{m_{2} R T}{V_{2}^{2}}\right)$.

With Eqs. (8) and (9), Eq. (12) can be rewritten as

$k_{\theta}=\left(\frac{3 \delta\left(a^{2}-b^{2}\right)}{2}\right)^{2}\left(\frac{m_{1} R T}{\left(V_{10}-A_{1} x+\frac{3 \delta\left(a^{2}-b^{2}\right) \theta}{2}\right)^{2}}+\frac{m_{2} R T}{\left(V_{20}+A_{2} x-\frac{3 \delta\left(a^{2}-b^{2}\right) \theta}{2}\right)^{2}}\right)$.

The ratio $r$ can be derived from the following relationship:

$\frac{3 \delta\left(a^{2}-b^{2}\right)}{2} d \theta=A_{1} d x$.

From this relationship, the ratio $r$ is defined as

$r \triangleq \frac{d x}{d \theta}=\frac{3 \delta\left(a^{2}-b^{2}\right)}{2 A_{1}}$.

\section{Control strategy}

\subsection{Problem statement}

The objective of the control problem is to design the control input $F_{m}$ that causes the endeffector $(\theta)$ to track a desired trajectory $\left(\theta_{d}\right)$. The first step is to linearize the map between the 
input $F_{m}$ and the actual output $\theta$, using the fact that SEA systems are feedback linearizable. With the state variables defined as $x_{1}=\theta, x_{2}=\dot{\theta}, x_{3}=x$, and $x_{4}=\dot{x}$, the equations of motion (6) are expressed in state-space form as

$\dot{x}_{1}=x_{2}$,

$\dot{x}_{2}=\left(k_{\theta}\left(x_{3} / r-x_{1}\right)+G\left(x_{1}\right)+F_{f 1}+D_{1}\right) / I$,

$\dot{x}_{3}=x_{4}$,

$\dot{x}_{4}=\left(-k_{x}\left(x_{3}-r x_{1}\right)+F_{f 2}+D_{2}+F_{m}\right) / B$

where $G \triangleq-m g h \sin x_{1}$.

Under the following nonlinear state coordinate transformation

$z_{1}=x_{1}$,

$z_{2}=x_{2}$,

$z_{3}=\left(k_{\theta}\left(x_{3} / r-x_{1}\right)+G\left(z_{1}\right)+F_{f 1}+D_{1}\right) / I$,

$z_{4}=\left(k_{\theta}\left(x_{4} / r-x_{2}\right)+\dot{G}\left(z_{1}\right)+\dot{F}_{f 1}+\dot{D}_{1}\right) / I$,

the model (16) can be written in terms of the new coordinates as

$\dot{z}_{1}=z_{2}$,

$\dot{z}_{2}=z_{3}$,

$\dot{z}_{3}=z_{4}$,

$\dot{z}_{4}=\alpha(\mathbf{z})+\beta F_{m}$,

where $\mathbf{z}=\left[\begin{array}{lll}z_{1} & z_{2} & z_{3}\end{array}\right]^{T}$,

$\alpha(\mathbf{z})=-\frac{k_{\theta}}{I} z_{3}-\frac{k_{x}}{B} z_{3}+\frac{1}{I}\left(\ddot{G}\left(z_{1}\right)+\ddot{F}_{f 1}+\ddot{D}_{1}\right)+\frac{k_{x}}{I B}\left(G\left(z_{1}\right)+F_{f 1}+D_{1}\right)+\frac{k_{\theta}}{r I B}\left(F_{f 2}+D_{2}\right)$,

$\beta=\frac{k_{\theta}}{r I B}$

\subsection{Controller design}

Continuous-time systems are typically controlled in digital implementation. A controller is discretized and implemented with sample and hold devices. Assuming that the state variables of the system are measured at sampling instants, a discrete-time controller is designed as delayed control as follows [40]:

$F_{m}(t)=F_{m d}\left(t_{k}\right)=F_{m d}(t-d(t)), t \in\left[t_{k}, t_{k+1}\right)$,

where $F_{m d}$ is a discrete-time control input, $0=t_{0}<t_{1}<\cdots<t_{k}<\cdots$ are the sampling instants, and $d(t)=t-t_{k} \leq d_{\max }=L$ (fixed sampling interval) which denotes a piecewise continuous delay with $\dot{d}(t)=1$.

From now on, a controller using the TDE technique is designed that moves the end-effector $\left(z_{1}\right)$ to the desired trajectory $\left(z_{d}\right)$, eliminating the need of identifying a system model (i.e., $\alpha, \beta$ in Eq. (18)). 
The last line of Eq. (18) can be rewritten, by introducing a constant $\bar{\beta}$, as follows: $\dot{z}_{4}=z_{1}^{(4)}=\alpha(\mathbf{z})+\beta F_{m d}(t-d(t))-\bar{\beta} F_{m d}(t-d(t))+\bar{\beta} F_{m d}(t-d(t))=H+\bar{\beta} F_{m d}(t-d(t))$,

$\mathbf{z}(\vartheta)=\varphi(\vartheta), \quad$ with $\vartheta \in\left[-d_{\max }, 0\right]$,

where $H \triangleq \alpha(\mathbf{z})+\beta F_{m d}(t-d(t))-\bar{\beta} F_{m d}(t-d(t)), \varphi \subseteq \mathbf{R}^{n}$ is the minimal information to obtain the solution $\mathbf{z}\left(t, \varphi, t_{0}\right)$.

The term $H$ contains system dynamics and all uncertainties. Originally, TDE-incorporated controllers are developed based on the assumption that the term $H$ is piecewise continuous since the system (6) is generally composed of continuous components. The assumption enables the term $H$ to be estimated; the current value of the term $H$ can be estimated with the previous-step control input and its corresponding measured output. In the proposed control, the estimation of the current value of the term $H$ can be achieved through the following estimator:

$\widehat{H}(t-d(t)) \triangleq z_{1}^{(4)}(t-d(t))-\bar{\beta} F_{m d}(t-d(t)-L)$.

The terms $z_{1}^{(4)}(t-d(t))$ and $F_{m d}(t-d(t)-L)$ are the latest measured state and the previous-step control input, respectively. The accuracy of TDE can be improved by reducing the difference between $t$ and $t_{k}$. This can be achieved by increasing the sampling frequency. Utilizing this approach, the control law is constructed as

$F_{m d}(t-d(t))=\frac{1}{\bar{\beta}}(-\widehat{H}(t-d(t))+v)=F_{m d}(t-d(t)-L)+\frac{1}{\bar{\beta}}\left(-z_{1}^{(4)}(t-d(t))+v\right)$.

The new control input, defined as $v$, determines the error dynamics of the system. The new input $v$ for position control can be designed as

$v=z_{d}^{(4)}(t-d(t))+c_{1} \dddot{e}(t-d(t))+c_{2} \ddot{e}(t-d(t))+c_{3} \dot{e}(t-d(t))+c_{4} e(t-d(t))$,

where $e(t) \triangleq z_{d}(t)-z_{1}(t)$ and $c_{1}, \ldots, c_{4}$ are design parameters.

\subsection{TDE error compensation}

We note that the control law does not guarantee asymptotic stability since $\widehat{H}(t) \neq H(t)$ (details are presented in an Appendix). In particular, when discontinuous components such as friction are included in the term $H$, the difference between the real value of $H$ and its estimate becomes significant. TDE error, an estimation error, arises from the difference. TDE error is involved in the convergence of the error dynamics. With TDE error defined as

$\varepsilon(t) \triangleq \widehat{H}(t-d(t))-H(t)=v-z_{1}^{(4)}(t)$,

the error dynamics of the proposed controller including TDE error is obtained by substituting Eq. (26) into Eq. (27) as follows:

$\varepsilon(t)=z_{d}^{(4)}(t-d(t))-z_{1}^{(4)}(t)+c_{1} \dddot{e}(t-d(t))+c_{2} \ddot{e}(t-d(t))+c_{3} \dot{e}(t-d(t))+c_{4} e(t-d(t))$. 
Note that the TDE error $\varepsilon$ causes the system output to deviate from the desired trajectory. In an effort to reduce the influence of $\varepsilon$ on the error dynamics and pursue asymptotic stability, a learning algorithm is adopted that estimates the TDE error $\varepsilon$. The update law is

$\widehat{\varepsilon}(t-d(t))=\widehat{\varepsilon}(t-d(t)-L)-\gamma \xi(t-d(t))$,

where $\gamma$ is a learning factor and $\xi$ denotes tracking error.

The tracking error can be designed as

$$
\begin{aligned}
\xi(t-d(t)) \triangleq & k_{1} e^{(4)}(t-d(t))+k_{2} \dddot{e}(t-d(t))+k_{3} \ddot{e}(t-d(t)) \\
& +k_{4} \dot{e}(t-d(t))+k_{5} e(t-d(t)),
\end{aligned}
$$

where $k_{1}, \ldots, k_{5}$ are gains.

The proposed control law considering the counteracting term $\widehat{\varepsilon}(t-d)$ is written as follows:

$$
\begin{aligned}
F_{m d}(t-d(t))= & F_{m d}(t-d(t)-L)+\bar{\beta}^{-1}\left[z_{d}^{(4)}(t-d(t))-z_{1}^{(4)}(t-d(t))+c_{1} \dddot{e}(t-d(t))\right. \\
& \left.+c_{2} \ddot{e}(t-d(t))+c_{3} \dot{e}(t-d(t))+c_{4} e(t-d(t))+\widehat{\varepsilon}(t-d(t))\right] .
\end{aligned}
$$

The position and its derivatives of the driven part need to be fed back to the control law including the TDE error compensation. As long as the sampling interval $L$ is sufficiently small and the learning algorithm estimates the TDE error $\varepsilon$ in a satisfactory manner, the target system can be asymptotically stabilized with the control input. The proof of the stability of the closed-loop system is presented in the Appendix.

\subsection{Measurement noise and remedies}

The proposed control requires the position state and its derivatives to be measured. For practical reasons, all required states are difficult to measure using sensors. Fortunately, the use of a relatively large value of $\bar{\beta}$ leads to the same effect as a 1st-order digital low-pass filter (LPF). One can rewrite the proposed control law (31) with a definition $w \triangleq e^{(4)}+c_{1} \dddot{e}+$ $c_{2} \ddot{e}+c_{3} \dot{e}+c_{4} e+\widehat{\varepsilon}$ as

$F_{m d}(t-d(t))=F_{m d}(t-d(t)-L)+\bar{\beta}^{-1} w$.

When the 1st-order LPF is injected into the control law, Eq. (32) can be rewritten as

$F_{m d}(t-d(t))=F_{m d}^{F}(t-d(t)-L)+\bar{\beta}^{-1} w$,

where $F_{m d}^{F}$ is the filtered control input.

Designing the 1st-order LPF with the cutoff frequency of $\lambda$ as

$F_{m d}^{F}(t-d(t))=\lambda L(1+1 \lambda L)^{-1} F_{m d}(t-d(t))+(1+1 \lambda L)^{-1} F_{m d}^{F}(t-d(t)-L)$,

and substituting Eq. (33) into Eq. (34) leads to

$F_{m d}^{F}(t-d(t))=F_{m d}^{F}(t-d(t)-L)+\bar{b}^{-1} w$,

where $\bar{b}^{-1}=\lambda L(1+1 \lambda L)^{-1} \bar{\beta}^{-1}$.

From the fact that Eqs. (32) and (35) are in the same form, it is concluded that increasing the value of $\bar{\beta}$ plays the same role as the 1st-order digital LPF. 


\section{Numerical validation}

A simulation study of the proposed control with the pneumatic system (Fig. 3) is conducted. The system parameters are presented in Table 1 , based on [8,9]. First, it is required to examine how the proposed control provides robustsness to uncertainties including unknown disturbances and measurement noise. Next the effect of the learning TDE error compensation is examined with the learning factor $\gamma$ varying. Through the simulation study, it is assumed that the position and acceleration of the driven part are measured using separate sensors and we obtain the velocity from the measured position state and jerk and its derivative from the acceleration state, respectively, through numerical differentiations.

The mismatched disturbance $D_{1}$ and matched disturbance $D_{2}$ are imposed as $0.61 \sin (60 t)$ and $\cos (60 t)$, respectively [41]. Friction $F_{f 1}, F_{f 2}$ are set as $0.02 \dot{q}_{1}+0.02 \operatorname{sgn}\left(\dot{q}_{1}\right) \mathrm{Nm}$ and $2 \dot{q}_{2}+2 \operatorname{sgn}\left(\dot{q}_{2}\right) \mathrm{N}$, respectively. The sampling time $L$ is $0.001 \mathrm{~s}$. The 4 th-order Runge-Kutta method is adopted to solve system dynamics with step size $0.0001 \mathrm{~s}$. The initial conditions of all system states are all set to 0 .

\subsection{Performance over uncertainties}

To investigate the capability of the proposed control in compensating for system dynamics and uncertainties, a controller proposed in [42] is selected as a comparison target. The control, which is based on feedback linearization, adopted an extended state observer (ESO) to estimate system dynamics and uncertainties.

The control gains $c_{1}, c_{2}, c_{3}$, and $c_{4}$ in the proposed control are determined by placing the poles at $\left(1+\tau_{c} s\right)^{4}$ with $\tau_{c}=1 / 15$. The gain $\bar{\beta}$ is located at 250 . We determine the control gains $k_{1}, k_{2}, k_{3}$, and $k_{4}$ in the ESO control by placing the same poles, i.e., $\left(1+\tau_{c} s\right)^{4}$ with $\tau_{c}=1 / 15$, to produce the same error dynamics. The observer gains $\beta_{1}, \beta_{2}, \beta_{3}, \beta_{4}$, and $\beta_{5}$ in the ESO control are obtained by placing the same poles, i.e., $\left(1+\tau_{o} s\right)^{5}$ with $\tau_{o}=$ $1 / 30$. The nominal value $b_{0}$ in the ESO control law is set to 150 while the real value of $b$ (in their denotation) is around 152. The learning counteraction is turned off through this comparison.

Table 1

Parameters of the pneumatic system.

\begin{tabular}{llll}
\hline Parameter & Symbol & Value & Unit \\
\hline Driven part inertia & $I$ & 0.04 & $\mathrm{kgm}^{2}$ \\
Gravitational torque & $m g h$ & 2 & $\mathrm{Nm}$ \\
Driving part inertia & $B$ & 1.5 & $\mathrm{~kg}$ \\
Driving cylinder bore & $A_{1}, A_{2}$ & $0.0011,0.0009$ & $\mathrm{~m}$ \\
Universal gas constant & $R$ & 287 & $\mathrm{~J} / \mathrm{kgK}$ \\
Temperature & $T$ & 293 & $\mathrm{~K}$ \\
Initial volume & $V_{10}, V_{20}$ & $\mathrm{~m}^{3}$ \\
Air mass & $m_{1}, m_{2}$ & 0.0003 & $\mathrm{~kg}$ \\
Air chamber thickness & $\delta$ & $3.6123 \times 10^{-04}$ & $\mathrm{~m}$ \\
Air chamber outer diameter & $a$ & 0.03 & $\mathrm{~m}$ \\
Air chamber inner diameter & $b$ & 0.04 & $\mathrm{~m}$ \\
\hline
\end{tabular}




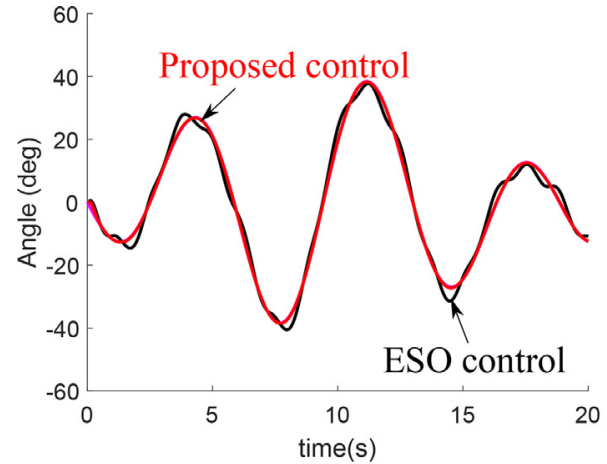

(a)

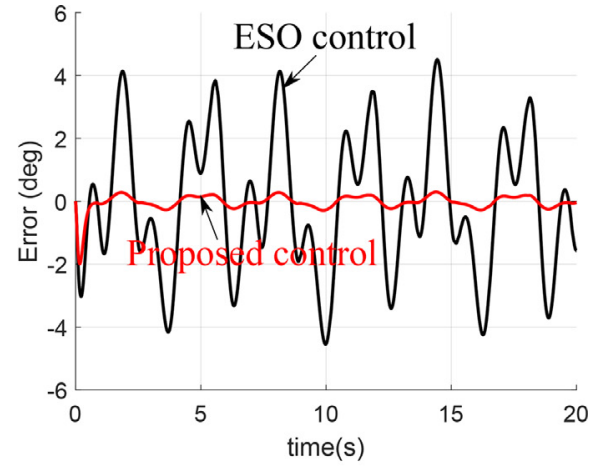

(b)

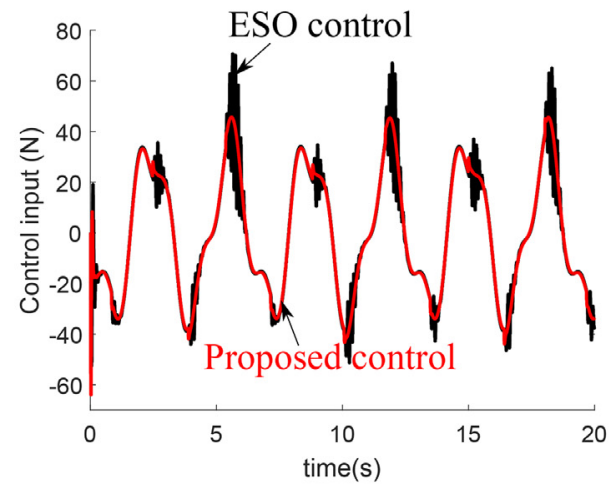

(c)

Fig. 4. Comparisons in performance of the proposed control with the ESO control (without measurement noise): (a) position (the desired trajectory (magenta line) is behind the red line), (b) position error and (c) control input.

Fig. 4 shows the comparisons in performance between the two controls. It is observed that the proposed control provides improved accuracy in tracking in comparison with the ESO control, under a similar degree of control input. This implies that system dynamics and uncertainties are efficiently estimated and compensated by TDE in comparison with the ESO.

Next a situation under measurement noise is considered. For position sensor noise, zeromean Gaussian noise with the standard deviation $\sigma=0.005^{2}$ is applied, and for acceleration sensor noise, zero-mean Gaussian noise with the standard deviation $\sigma=0.01^{2}$ is applied.

Fig. 5 displays comparisons in tracking performance under measurement noise; the figures on the left column are the results of the proposed control, whereas the figures on the right column are the results of the ESO control. In comparison with the ESO control that uses an observer, it is observed that the proposed control is less affected by measurement noise from the fact that its control input is less contaminated by the noise. Note that the proposed control law contains an equivalent low-pass filter. The tracking performance does not show a notable change in the presence of noise. 
JID: FI

D. Kim and J. Lee

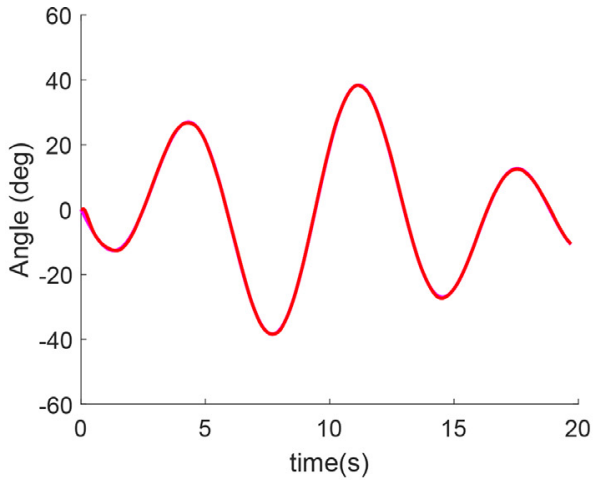

(a)

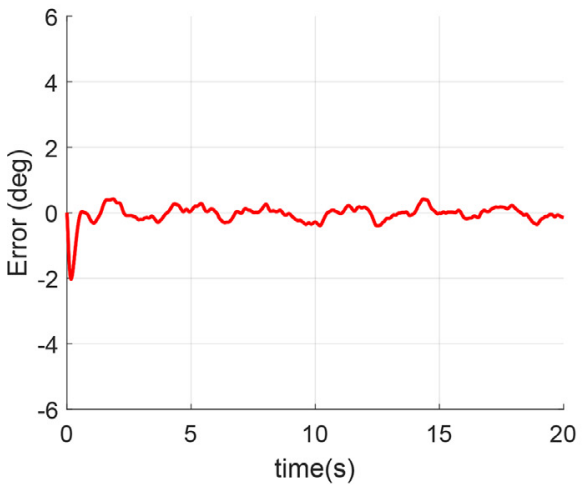

(c)

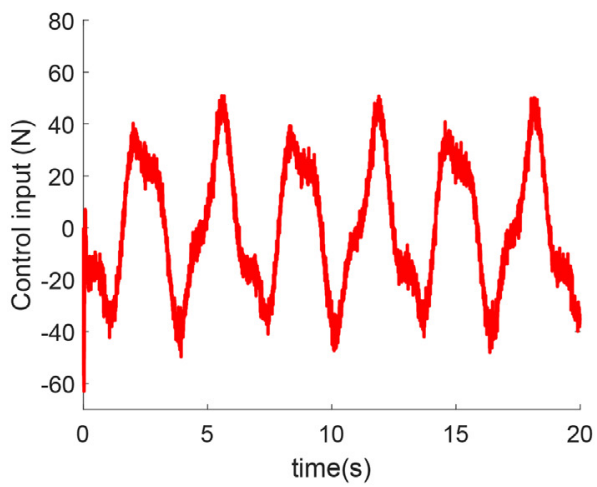

(e)
Journal of the Franklin Institute $x x x(x x x x) x x x$

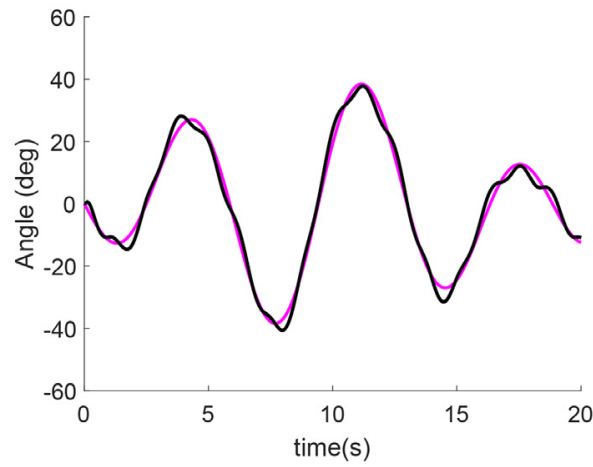

(b)

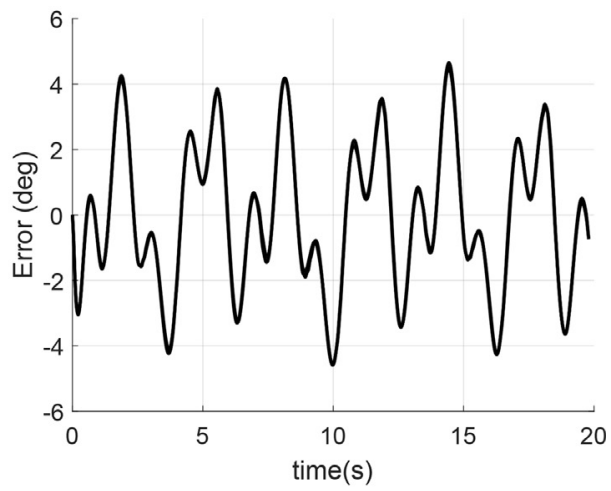

(d)

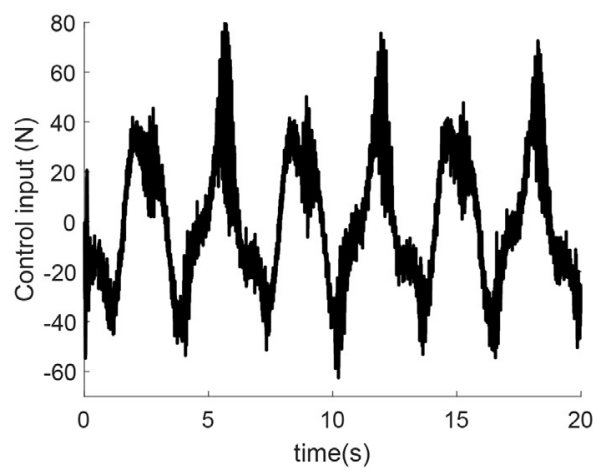

(f)

Fig. 5. Comparisons in performance of the proposed control (red line) with the ESO control (black line) under measurement noise: (a), (b) position, (c), (d) position error, and (e), (f) control input. The magenta lines in the position plots indicate the desired trajectory. (For interpretation of the references to color in this figure legend, the reader is referred to the web version of this article.) 


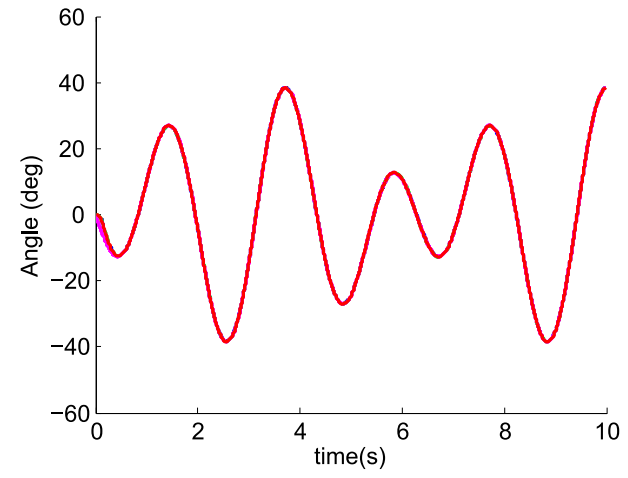

(a)

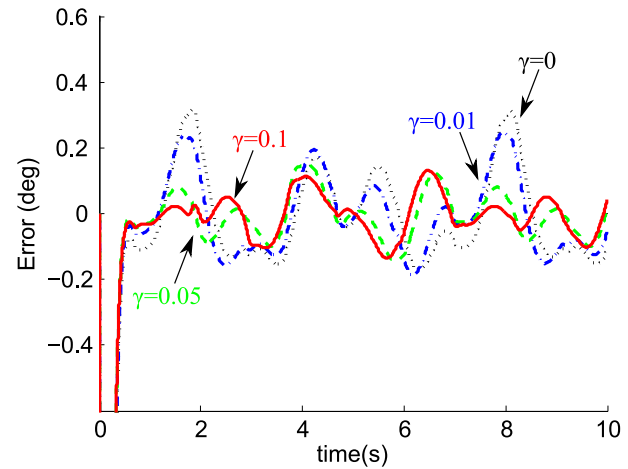

(b)

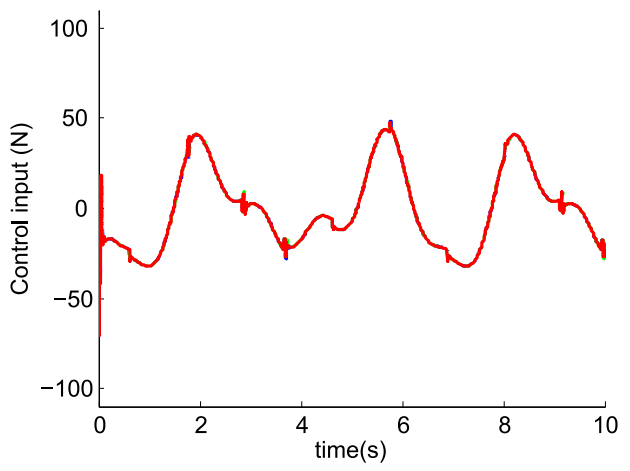

(c)

Fig. 6. Performance of the proposed control with different values of the learning factor $\gamma$ : (a) position (four curves overlap), (b) position error, and (c) control input (four curves overlap). The magenta line in the position plot indicates the desired trajectory.

\subsection{Effect of TDE error compensation}

The effect of TDE error compensation using the learning algorithm is examined with four different values of the learning factor $\gamma: 0,0.01,0.05$, and 0.1 . The control gains $c_{1}, c_{2}, c_{3}$, and $c_{4}$ in the proposed control are determined by placing the poles at $\left(1+\tau_{c} s\right)^{4}$ with $\tau_{c}=1 / 20$. The gains $k_{1}, k_{2}, k_{3}, k_{4}$, and $k_{5}$ in Eq. (30) are selected by placing the same poles $\left(\left(1+\tau_{c p} s\right)^{5}\right.$ with $\left.\tau_{o}=1 / 20\right)$. All unstructured uncertainties are imposed. Measurement noise is not considered at this time to test the effect of TDE error compensation.

Fig. 6 shows that a greater value of $\gamma$ provides more accuracy in tracking. This suggests that the term $\widehat{\varepsilon}$ in the control law that is introduced to counteract TDE error plays a role in cancelling out TDE error that influences the convergence of the error dynamics. This accordingly leads to reduced position error. Counteracting TDE error does not require a significant increase in control input. 


\section{Conclusion}

With the need of controlling the position of an arm exoskeleton actuated by a motor system through pneumatic circuits, a model-free and robust controller by incorporating the TDE technique and learning compensation has been developed. The TDE technique compensates for system dynamics and all kinds of uncertainties with no significant computation load. The learning compensation leads to asymptotic stability by cancelling out TDE error. The controller is designed and its closed-loop stability is analyzed in a sampled-data environment, redefining the causality about the control command and its output in TDE from a practical aspect. Numerical simulation through a comparative study, the proposed controller achieves satisfactory control performance in the presence of mismatched uncertainties as well as matched uncertainties. And it was founded that the learning counteraction for TDE error further reduces position tracking error.

\section{Declaration of Competing Interest}

The authors declare that the research was conducted in the absence of any commercial or financial relationships that could be construed as a potential conflict of interest.

\section{Acknowledgment}

The authors express our gratitude to Drs. Maolin Jin and Gun-Rae Cho at the Korea Institute of Robot and Convergence for their advice for this study. This publication was in part made possible by the University of Maryland Baltimore Institute for Clinical and Translational Research (ICTR) which is funded in part by Grant Number TL1 TR003100 from the National Center for Advancing Translational Sciences (NCATS) a component of the National Institutes of Health (NIH), and NIH Roadmap for Medical Research. Its contents are solely the responsibility of the authors and do not necessarily represent the official view of the University of Maryland Baltimore ICTR, NCATS or NIH.

\section{Appendix A}

\section{A1. Stability with TDE only}

Lemma 1. If 1) $\widehat{H}=H$, 2) $d_{\max }(=L)$ is sufficiently small, and 3) $\mathbf{A}_{0}+\mathbf{A}_{1}$ is a Hurwitz matrix, then the system (6) with control input (25) is asymptotically stable.

Proof. Assuming that $z_{d}^{(4)}(t) \approx z_{d}^{(4)}(t-d(t))$, the closed-loop dynamics can be expressed as

$$
\begin{aligned}
e^{(4)}(t) & =-c_{1} \dddot{e}(t-d(t))-c_{2} \ddot{e}(t-d(t))-c_{3} \dot{e}(t-d(t)) \\
& -c_{4} e(t-d(t)) .
\end{aligned}
$$

With introducing the matrices $\mathbf{A}_{0}, \mathbf{A}_{1} \in \mathbf{R}^{4 \times 4}$, Eq. (36) is rewritten in a matrix form as follows:

$\dot{\mathbf{e}}(t)=\mathbf{A}_{0} \mathbf{e}(t)+\mathbf{A}_{1} \mathbf{e}(t-d(t))$, 
$\mathbf{e}(\vartheta)=\varphi(\vartheta), \quad$ with $\vartheta \in\left[-d_{\max }, 0\right]$,

where

$\mathbf{A}_{0}=\left[\begin{array}{llll}0 & 1 & 0 & 0 \\ 0 & 0 & 1 & 0 \\ 0 & 0 & 0 & 1 \\ 0 & 0 & 0 & 0\end{array}\right]$,

$\mathbf{A}_{1}=\left[\begin{array}{cccc}0 & 0 & 0 & 0 \\ 0 & 0 & 0 & 0 \\ 0 & 0 & 0 & 0 \\ -c_{4} & -c_{3} & -c_{2} & -c_{1}\end{array}\right]$,

$\mathbf{e}(t)=\left[\begin{array}{llll}e(t) & \dot{e}(t) & \ddot{e}(t) & \dddot{e}(t)\end{array}\right]^{T}$.

One can express Eq. (37) in a descriptor form as follows:

$\dot{\mathbf{e}}(t)=\mathbf{y}(t)$,

$\mathbf{0}=-\mathbf{y}(t)+\left(\mathbf{A}_{0}+\mathbf{A}_{1}\right) \mathbf{e}(t)-\mathbf{A}_{1} \int_{t-d(t)}^{t} \mathbf{y}(s) d s$.

Again, Eq. (37) can be written in an extended form as

$F \dot{\overline{\mathbf{e}}}(t)=\left[\begin{array}{c}\dot{\mathbf{e}}(t) \\ \mathbf{0}\end{array}\right]$

$=\left[\begin{array}{cc}\mathbf{0} & \mathbf{I} \\ \mathbf{A}_{0}+\mathbf{A}_{1} & -\mathbf{I}\end{array}\right] \overline{\mathbf{e}}(t)-\left[\begin{array}{c}\mathbf{0} \\ \mathbf{A}_{1}\end{array}\right] \int_{t-d(t)}^{t} \mathbf{y}(s) d s$,

where $\overline{\mathbf{e}}(t)=\left[\begin{array}{ll}\mathbf{e}^{T}(t) & \mathbf{y}^{T}(t)\end{array}\right]^{T}$ and $\mathbf{F}=\operatorname{diag}[\mathbf{I}, \mathbf{0}]$.

Next, a Lyapunov-Krasovskii functional with positive $4 \times 4$ matrices $\mathbf{P}_{1}, \mathbf{P}_{2}, \mathbf{P}_{3}, \mathbf{Y}_{1}, \mathbf{Y}_{2}, \mathbf{Z}_{1}, \mathbf{Z}_{2}, \mathbf{Z}_{3}, \mathbf{R}$, and $\mathbf{S}$ is employed as follows:

$W_{1}(t) \triangleq \overline{\mathbf{e}}^{T}(t) \mathbf{F P} \overline{\mathbf{e}}(t)+\int_{-L}^{0} \int_{t+\varrho}^{t} \mathbf{y}^{T}(s) \mathbf{R y}(s) d s d \varrho$,

where

$\mathbf{P} \triangleq\left[\begin{array}{cc}\mathbf{P}_{1} & \mathbf{0} \\ \mathbf{P}_{2} & \mathbf{P}_{3}\end{array}\right], \quad \mathbf{F P}=\mathbf{P}^{T} \mathbf{F} \geq 0$.

Differentiating $W_{1}$ along the trajectories of Eq. (37) leads to (refer to [43] for details)

$\dot{W}_{1}(t) \leq \overline{\mathbf{e}}^{T}(t) \boldsymbol{\Psi} \overline{\mathbf{e}}(t)-\int_{t-L}^{t} \mathbf{y}^{T}(s) \mathbf{R y}(s) d s+\eta(t)$,

where

$\eta(t) \leq \int_{t-L}^{t}\left[\begin{array}{ll}\mathbf{y}^{T}(s) & \left.\overline{\mathbf{e}}^{T}(t)\right][ \\ \mathbf{Y}^{T}-\mathbf{P}^{T}\left[\begin{array}{c}\mathbf{0} \\ \mathbf{A}_{1}\end{array}\right] & \mathbf{Y}-\left[\begin{array}{cc}\mathbf{0} & \mathbf{A}_{1}^{T}\end{array}\right] \mathbf{P} \\ \mathbf{Z}\end{array}\right]\left[\begin{array}{l}\mathbf{y}(s) \\ \overline{\mathbf{e}}(t)\end{array}\right] d s$ 


$$
\begin{aligned}
= & \int_{t-L}^{t} \mathbf{y}^{T}(s) \mathbf{R y}(s) d s+2 \int_{t-L}^{t} \mathbf{y}^{T}(s)\left(\mathbf{Y}-\left[\begin{array}{ll}
\mathbf{0} & \mathbf{A}_{1}^{T}
\end{array}\right] \mathbf{P}\right) \overline{\mathbf{e}}(t) d s+\int_{t-L}^{t} \overline{\mathbf{e}}^{T}(t) \mathbf{Z} \overline{\mathbf{e}}(t) d s \\
= & \int_{t-L}^{t} \mathbf{y}^{T}(s) \mathbf{R y}(s) d s+2 \int_{t-L}^{t} \dot{\mathbf{e}}^{T}(s)\left(\mathbf{Y}-\left[\begin{array}{ll}
\mathbf{0} & \mathbf{A}_{1}^{T}
\end{array}\right] \mathbf{P}\right) \overline{\mathbf{e}}(t) d s+L \overline{\mathbf{e}}^{T}(t) \mathbf{Z} \overline{\mathbf{e}}(t) \\
\leq & \int_{t-L}^{t} \mathbf{y}^{T}(s) \mathbf{R y}(s) d s+2 \mathbf{e}^{T}(t)\left(\mathbf{Y}-\left[\begin{array}{ll}
\mathbf{0} & \mathbf{A}_{1}^{T}
\end{array}\right] \mathbf{P}\right) \overline{\mathbf{e}}(t)-2 \mathbf{e}^{T}(t-L)\left(\mathbf{Y}-\left[\begin{array}{ll}
\mathbf{0} & \mathbf{A}_{1}^{T}
\end{array}\right] \mathbf{P}\right) \overline{\mathbf{e}}(t) \\
& +L \overline{\mathbf{e}}^{T}(t) \mathbf{Z} \overline{\mathbf{e}}(t),
\end{aligned}
$$

$$
\begin{aligned}
\boldsymbol{\Psi} \triangleq & \mathbf{P}^{T}\left[\begin{array}{cc}
\mathbf{0} & \mathbf{I} \\
\mathbf{A}_{0} & -\mathbf{I}
\end{array}\right]+\left[\begin{array}{cc}
\mathbf{0} & \mathbf{I} \\
\mathbf{A}_{0} & -\mathbf{I}
\end{array}\right]{ }^{T} \mathbf{P}+d_{\max } \mathbf{Z} \\
& +\left[\begin{array}{cc}
\mathbf{0} & \mathbf{0} \\
\mathbf{0} & d_{\max } \mathbf{R}
\end{array}\right],
\end{aligned}
$$

and

$\mathbf{Z} \triangleq\left[\begin{array}{ll}\mathbf{Z}_{1} & \mathbf{Z}_{2} \\ & \mathbf{Z}_{3}\end{array}\right], \mathbf{Y} \triangleq\left[\begin{array}{ll}\mathbf{Y}_{1} & \mathbf{Y}_{2}\end{array}\right]$

Then, Eq. (47) can be rewritten as

$$
\begin{aligned}
\dot{W}_{1}(t) \leq & \overline{\mathbf{e}}^{T}(t) \Psi \overline{\mathbf{e}}(t)+2 \mathbf{e}^{T}(t)\left(\mathbf{Y}-\left[\begin{array}{ll}
\mathbf{0} & \mathbf{A}_{1}^{T}
\end{array}\right] \mathbf{P}\right) \overline{\mathbf{e}}(t)-2 \mathbf{e}^{T}(t-d(t))\left(\mathbf{Y}-\left[\begin{array}{ll}
\mathbf{0} & \mathbf{A}_{1}^{T}
\end{array}\right] \mathbf{P}\right) \overline{\mathbf{e}}(t) \\
& +L \overline{\mathbf{e}}^{T}(t) \mathbf{Z} \overline{\mathbf{e}}(t)
\end{aligned}
$$

Under the the conditions $\mathbf{Y}=\left[\begin{array}{ll}\mathbf{0} & \mathbf{A}_{1}^{T}\end{array}\right] \mathbf{P}$ and $\mathbf{R}$ and $\mathbf{Z} \rightarrow \mathbf{0}$ (and $d_{\max }$ is assumed sufficiently small), Eq. (51) can be rewritten as

$\dot{W}_{1}(t) \leq \overline{\mathbf{e}}^{T}(t) \overline{\boldsymbol{\Gamma}} \overline{\mathbf{e}}(t)$,

where

$$
\begin{gathered}
\overline{\boldsymbol{\Gamma}} \triangleq\left[\begin{array}{cc}
\boldsymbol{\phi} & \mathbf{P}_{1}-\mathbf{P}_{2}^{T}+\left(\mathbf{A}_{0}+\mathbf{A}_{1}\right)^{T} \mathbf{P}_{3} \\
-\mathbf{P}_{3}-\mathbf{P}_{3}^{T}
\end{array}\right], \\
\boldsymbol{\phi} \triangleq \quad \mathbf{P}_{2}^{T}\left(\mathbf{A}_{0}+\mathbf{A}_{1}\right)+\left(\mathbf{A}_{0}+\mathbf{A}_{1}\right)^{T} \mathbf{P}_{2} .
\end{gathered}
$$

If $\mathbf{P}_{1}=\mathbf{P}_{2}, \mathbf{P}_{3} \rightarrow \mathbf{0}$ and $\boldsymbol{\phi}<0$, then $\dot{W}_{1}(t)<0$. This result indicates that the system (6) with control input (25) is asymptotically stable. Note that we assume that Lemma 1 has been constructed with the condition the ideal assumption $(\widehat{H}=H)$ which is unrealistic. If the ideal assumption $(\widehat{H}=H)$ is broken, asymptotic stability cannot be guaranteed. Also, it is possible that performance degrades.

A2. Stability with TDE and the learning-based counteraction

Lemma 2. The system (6) with control input (31) is asymptotically stable if 1) $d_{\max }(=L)$ is sufficiently small, 2) $\mathbf{A}_{0}+\mathbf{A}_{1}$ is a Hurwitz matrix, and 3) $\xi$ is designed as follows:

$$
\begin{aligned}
\xi(t) & =2 a\left[\mathbf{e}^{T}(t) \dot{\mathbf{e}}^{T}(t)\right] \mathbf{D} \\
& =2 a\left(P_{3}^{4,4} e^{(4)}(t)+\left(P_{2}^{4,4}+P_{3}^{3,4}\right) \dddot{e}(t)+\left(P_{2}^{3,4}+P_{3}^{2,4}\right) \ddot{e}(t)\right.
\end{aligned}
$$


JID: FI

D. Kim and J. Lee
[m1+;November 24, 2020;2:29]

Journal of the Franklin Institute $x x x(x x x x) x x x$

$$
\left.+\left(P_{2}^{2,4}+P_{3}^{1,4}\right) \dot{e}(t)+P_{2}^{1,4} e(t)\right),
$$

where

$\mathbf{D} \triangleq\left[\begin{array}{llllll}P_{2}^{1,4} & \cdots & P_{2}^{4,4} & P_{3}^{1,4} & \cdots & P_{3}^{4,4}\end{array}\right]^{T}$,

with $P_{2}^{i, j}, P_{3}^{i, j}$ denoting the $(i, j)$ th element of $\mathbf{P}_{2}, \mathbf{P}_{3}$, respectively. $P_{2}^{i, j}, P_{3}^{i, j}$ are selected such that the matrices $\mathbf{P}_{2}, \mathbf{P}_{3}$ remain positive.

Proof. The closed-loop dynamics can be expressed in a matrix form as

$\dot{\mathbf{e}}(t)=\mathbf{A}_{0} \mathbf{e}(t)+\mathbf{A}_{1} \mathbf{e}(t-d(t))+\mathbf{E}(t)$,

where

$\mathbf{E}(t)=\left[\begin{array}{llll}0 & 0 & 0 & \widetilde{\varepsilon}\end{array}\right]^{T}, \quad(\widetilde{\varepsilon}(t) \triangleq \varepsilon(t)-\widehat{\varepsilon}(t-d(t)))$.

In a similar way to Eq. (45), Eq. (57) is expressed in an extended form as

$$
\begin{aligned}
& \mathbf{F} \dot{\overrightarrow{\mathbf{e}}}(t)=\left[\begin{array}{c}
\dot{\mathbf{e}}(t) \\
\mathbf{0}
\end{array}\right] \\
& =\left[\begin{array}{cc}
\mathbf{0} & \mathbf{I} \\
\mathbf{A}_{0}+\mathbf{A}_{1} & -\mathbf{I}
\end{array}\right] \overline{\mathbf{e}}(t)-\left[\begin{array}{c}
\mathbf{0} \\
\mathbf{A}_{1}
\end{array}\right] \int_{t-d(t)}^{t} \mathbf{y}(s) d s \\
& +\left[\begin{array}{c}
\mathbf{0} \\
\mathbf{E}(t)
\end{array}\right] .
\end{aligned}
$$

The following Lyapunov-Krasovskii functional can be designed:

$W(t) \triangleq W_{1}(t)+W_{2}(t)=W_{1}(t)+m \int_{t-L}^{t} \frac{1}{2} \widetilde{\varepsilon}^{2}(s) d s$,

where $m>0$.

Differentiating $W_{1}$ along the trajectories of Eq. (57) leads to

$$
\begin{aligned}
\dot{W}_{1}(t) \leq & \overline{\mathbf{e}}^{T}(t) \Psi \overline{\mathbf{e}}(t)+2 \mathbf{e}^{T}(t)\left(\mathbf{Y}-\left[\begin{array}{ll}
\mathbf{0} & \mathbf{A}_{1}^{T}
\end{array}\right] \mathbf{P}\right) \overline{\mathbf{e}}(t) \\
& -2 \mathbf{e}^{T}(t-d(t))\left(\mathbf{Y}-\left[\begin{array}{ll}
\mathbf{0} & \mathbf{A}_{1}^{T}
\end{array}\right] \mathbf{P}\right) \overline{\mathbf{e}}(t)+L \overline{\mathbf{e}}^{T}(t) \mathbf{Z} \overline{\mathbf{e}}(t) \\
& +2 \overline{\mathbf{e}}^{T}(t) \mathbf{P}^{T}\left[\begin{array}{c}
\mathbf{0} \\
\mathbf{E}(t)
\end{array}\right] .
\end{aligned}
$$

Next, $W_{2}$ can be differentiated as

$$
\begin{aligned}
\dot{W}_{2}(t) & =\frac{m}{2}\left(\widetilde{\varepsilon}^{2}(t)-\widetilde{\varepsilon}^{2}(t-L)\right) \\
& =\frac{m}{2}(\widetilde{\varepsilon}(t)-\widetilde{\varepsilon}(t-L))(\widetilde{\varepsilon}(t)+\widetilde{\varepsilon}(t-L)) \\
& =\frac{m}{2} \bar{\varepsilon}(t)(2 \widetilde{\varepsilon}(t)-\bar{\varepsilon}(t)) \\
& =m \bar{\varepsilon}(t) \widetilde{\varepsilon}(t)-\frac{m}{2} \bar{\varepsilon}^{2}(t),
\end{aligned}
$$

where $\bar{\varepsilon}(t) \triangleq \widetilde{\varepsilon}(t)-\widetilde{\varepsilon}(t-L)$. 
$W_{2}$ can be rewritten as follows, with the relationship $\xi(t-d(t))=\frac{1}{\gamma}(\widehat{\varepsilon}(t-d(t))-\widehat{\varepsilon}(t-$ $d(t-L)-L))$ from Eq. (29):

$$
\begin{aligned}
\dot{W}_{2}(t) & \leq m \bar{\varepsilon}(t) \widetilde{\varepsilon}(t) \\
& =m((\varepsilon(t)-\varepsilon(t-L))-(\widehat{\varepsilon}(t-d(t))-\widehat{\varepsilon}(t-d(t-L)-L))) \widetilde{\varepsilon}(t) \\
& =m((\varepsilon(t)-\varepsilon(t-L)) \widetilde{\varepsilon}(t)-\gamma \xi(t-d(t)) \widetilde{\varepsilon}(t)) \\
& =m((\varepsilon(t)-\varepsilon(t-L)) \widetilde{\varepsilon}(t)-\gamma \xi(t) \widetilde{\varepsilon}(t)+\gamma(\xi(t)-\xi(t-d(t))) \widetilde{\varepsilon}(t)) .
\end{aligned}
$$

The summation of the above two Lyapunov functionals $\left(W \triangleq W_{1}+W_{2}\right)$ can be reduced to

$$
\begin{aligned}
\dot{W}(t) \leq & \overline{\mathbf{e}}^{T}(t) \overline{\boldsymbol{\Gamma}} \overline{\mathbf{e}}(t)-(1-\dot{d}(t)) \mathbf{e}^{T}(t-d(t)) S \mathbf{e}(t-d(t)) \\
& +2 \mathbf{e}^{T}(t)\left(\mathbf{Y}-\left[\begin{array}{ll}
\mathbf{0} & \mathbf{A}_{1}^{T}
\end{array}\right] \mathbf{P}\right) \overline{\mathbf{e}}(t) \\
& -2 \mathbf{e}^{T}(t-d(t))\left(\mathbf{Y}-\left[\begin{array}{ll}
\mathbf{0} & \mathbf{A}_{1}^{T}
\end{array}\right] \mathbf{P}\right) \overline{\mathbf{e}}(t) \\
& +L \overline{\mathbf{e}}^{T}(t) \mathbf{Z} \overline{\mathbf{e}}(t)+2 \overline{\mathbf{e}}^{T}(t) \mathbf{P}^{T}\left[\begin{array}{c}
\mathbf{0} \\
\mathbf{E}(t)
\end{array}\right]-m \gamma \xi(t) \widetilde{\varepsilon}(t) \\
& +m(\varepsilon(t)-\varepsilon(t-L)) \widetilde{\varepsilon}(t)+m \gamma(\xi(t)-\xi(t-d(t))) \widetilde{\varepsilon}(t),
\end{aligned}
$$

where the matrix $\overline{\boldsymbol{\Gamma}}$ was defined in Eq. (54).

As in Lemma 1 , if $d_{\max }(=L)$ is assumed sufficiently small and $\mathbf{Y}=\left[\begin{array}{ll}\mathbf{0} & \mathbf{A}_{1}^{T}\end{array}\right] \mathbf{P}, \mathbf{R}$ and $\mathbf{Z}$ $\rightarrow 0$, Eq. (65) is reduced to

$$
\begin{aligned}
\dot{W}(t) \leq & \overline{\mathbf{e}}^{T}(t)^{T} \overline{\boldsymbol{\Gamma}} \overline{\mathbf{e}}(t)+2 \overline{\mathbf{e}}^{T}(t) \mathbf{P}^{T}\left[\begin{array}{c}
\mathbf{0} \\
\mathbf{E}(t)
\end{array}\right]-m \gamma \xi(t) \widetilde{\varepsilon}(t) \\
& +m(\varepsilon(t)-\varepsilon(t-L)) \widetilde{\varepsilon}(t)+m \gamma(\xi(t)-\xi(t-d(t))) \widetilde{\varepsilon}(t) .
\end{aligned}
$$

The last two terms in the above equation becomes negligible:

- $m(\varepsilon(t)-\varepsilon(t-L)) \widetilde{\varepsilon}(t) \approx 0$ when $m \rightarrow 0$ and the TDE error estimation error $\widetilde{\varepsilon}(t) \rightarrow 0$.

- $m \gamma(\xi(t)-\xi(t-d(t))) \widetilde{\varepsilon}(t) \approx 0$ when $d(t)$ is sufficiently small and the TDE error estimation error $\widetilde{\varepsilon}(t) \rightarrow 0$.

Eliminating these negligible terms from Eq. (66), we have

$\dot{W}(t) \leq \overline{\mathbf{e}}^{T}(t)^{T} \boldsymbol{\Gamma} \overline{\mathbf{e}}(t)+2 \overline{\mathbf{e}}^{T}(t) \mathbf{P}^{T}\left[\begin{array}{c}\mathbf{0} \\ \mathbf{E}(t)\end{array}\right]-m \gamma \xi(t) \widetilde{\varepsilon}(t)$.

Based on Lemma 1, the matrix $\overline{\boldsymbol{\Gamma}}$ is negative if $d_{\max }(=L)$ is sufficiently small and $\mathbf{A}_{0}+\mathbf{A}_{1}$ is Hurwitz.

The middle term on the right-hand side of Eq. (67) can be rearranged as

$$
2 \overline{\mathbf{e}}^{T}(t) \mathbf{P}^{T}\left[\begin{array}{c}
\mathbf{0} \\
\mathbf{E}(t)
\end{array}\right]=2 \overline{\mathbf{e}}^{T}(t)\left[\begin{array}{c}
\mathbf{P}_{2}^{T} \mathbf{E}(t) \\
\mathbf{P}_{3}^{T} \mathbf{E}(t)
\end{array}\right]=2 \overline{\mathbf{e}}^{T}(t) \mathbf{D} \widetilde{\varepsilon}(t),
$$

with the matrix $\mathbf{D}$ defined in Eq. (56).

If $\xi(t)$ is designed such that

$$
\xi(t)=\frac{2}{m \gamma} \overline{\mathbf{e}}^{T}(t) \mathbf{D},
$$

as long as the matrices $\mathbf{P}_{2}, \mathbf{P}_{3}$ remain positive, one can obtain $\dot{W}(t) \leq \overline{\mathbf{e}}^{T}(t)^{T} \boldsymbol{\Gamma} \overline{\mathbf{e}}(t)$. 
The condition $\overline{\boldsymbol{\Gamma}}<0$ leads to that $\dot{W}(t)<0$. Therefore, the closed-loop system is globally asymptotically stable.

Remark 1. The gains $k_{1}, \ldots, k_{5}$ in Eq. (30) correspond to the coefficients of Eq. (55), respectively. The gains $k_{1}, \ldots, k_{5}$ need to be selected that keep $\mathbf{P}_{2}, \mathbf{P}_{3}$ positive to achieve asymptotic stability.

Remark 2. The gain $\bar{\beta}$ does not influence stability theoretically, which is in accordance with [31]. Due to practical limitations including the actuator output limit and measurement noise, it is necessary to avoid setting the gain to a high value.

\section{References}

[1] G.A. Pratt, M.M. Williamson, Series elastic actuators, in: Proceedings of the 1995 IEEE/RSJ International Conference on Intelligent Robots and Systems 95. 'Human Robot Interaction and Cooperative Robots', 1, IEEE, 1995, pp. 399-406.

[2] M. Zinn, O. Khatib, B. Roth, J.K. Salisbury, Playing it safe [human-friendly robots], Robot. Autom. Mag. IEEE 11 (2) (2004) 12-21.

[3] H. Vallery, J. Veneman, E. Van Asseldonk, R. Ekkelenkamp, M. Buss, H. Van Der Kooij, Compliant actuation of rehabilitation robots, Robot. Autom. Mag. IEEE 15 (3) (2008) 60-69.

[4] K. Kong, J. Bae, M. Tomizuka, A compact rotary series elastic actuator for human assistive systems, Mechatron. IEEE/ASME Trans. 17 (2) (2012) 288-297.

[5] D. Accoto, G. Carpino, F. Sergi, N.L. Tagliamonte, L. Zollo, E. Guglielmelli, Design and characterization of a novel high-power series elastic actuator for a lower limb robotic orthosis, Int. J. Adv. Robot. Syst. 10 (10) (2013) 359 .

[6] C. Fitzgerald, Developing baxter, in: Proceedings of the 2013 IEEE International Conference on Technologies for Practical Robot Applications (TePRA), IEEE, 2013, pp. 1-6.

[7] H. Yu, S. Huang, G. Chen, Y. Pan, Z. Guo, Human-robot interaction control of rehabilitation robots with series elastic actuators, Robot. IEEE Trans. 31 (5) (2015) 1089-1100.

[8] R.B. Gillespie, D. Kim, J.M. Suchoski, B. Yu, J.D. Brown, Series elasticity for free free-space motion for free, in: Proceedings of the Haptics Symposium (HAPTICS), 2014 IEEE, IEEE, 2014, pp. 609-615.

[9] D. Kim, Compliant motion control for a compliant rehabilitation system, in: Proceedings of the 2015 IEEE International Conference on Rehabilitation Robotics (ICORR), IEEE, 2015, pp. 422-427.

[10] D. Kim, Design and analysis of an origami-structured actuator for backdriveability and power amplification, IEEE/ASME Trans. Mechatron. 24 (3) (2019) 1002-1010.

[11] R.H. Cannon, D.E. Rosenthal, Experiments in control of flexible structures with noncolocated sensors and actuators, J. Guid. Control Dyn. 7 (5) (1984) 546-553.

[12] P. Tomei, A simple pd controller for robots with elastic joints, Autom. Control IEEE Trans. 36 (10) (1991) 1208-1213.

[13] A. De Luca, B. Siciliano, L. Zollo, Pd control with on-line gravity compensation for robots with elastic joints: theory and experiments, Automatica 41 (10) (2005) 1809-1819.

[14] A. Albu-Schäffer, C. Ott, G. Hirzinger, A unified passivity-based control framework for position, torque and impedance control of flexible joint robots, Int. J. Robot. Res. 26 (1) (2007) 23-39.

[15] V. Zeman, R. Patel, K. Khorasani, Control of a flexible-joint robot using neural networks, Control Syst. Technol. IEEE Trans. 5 (4) (1997) 453-462.

[16] A.-C. Huang, Y.-C. Chen, Adaptive sliding control for single-link flexible-joint robot with mismatched uncertainties, Control Syst. Technol. IEEE Trans. 12 (5) (2004) 770-775.

[17] M.-C. Chien, A.-C. Huang, Adaptive control for flexible-joint electrically driven robot with time-varying uncertainties, Ind. Electron. IEEE Trans. 54 (2) (2007) 1032-1038.

[18] S. Ulrich, J.Z. Sasiadek, I. Barkana, Modeling and direct adaptive control of a flexible-joint manipulator, J. Guid. Control Dyn. 35 (1) (2012) 25-39.

[19] Y. Li, S. Tong, T. Li, Fuzzy adaptive dynamic surface control for a single-link flexible-joint robot, Nonlinear Dyn. 70 (3) (2012) 2035-2048.

[20] H.-M. Yen, T.-H. S. Li, Y.-C. Chang, Adaptive neural network based tracking control for electrically driven flexible-joint robots without velocity measurements, Comput. Math. Appl. 64 (5) (2012) 1022-1032. 
[21] M.A. Ahmad, M.Z.M. Tumari, A.N.K. Nasir, Composite fuzzy logic control approach to a flexible joint manipulator, Int. J. Adv. Robot. Syst. 10 (1) (2013) 58.

[22] C.T. Kiang, A. Spowage, C.K. Yoong, Review of control and sensor system of flexible manipulator, J. Intell. Robot. Syst. 77 (1) (2015) 187-213.

[23] K. Youcef-Toumi, O. Ito, A time delay controller for systems with unknown dynamics, in: Proceedings of the American Control Conference, 1988, IEEE, 1988, pp. 904-913.

[24] T.S. Hsia, T. Lasky, Z. Guo, Robust independent joint controller design for industrial robot manipulators, Ind. Electron. IEEE Trans. 38 (1) (1991) 21-25.

[25] M. Jin, S.H. Kang, P.H. Chang, Robust compliant motion control of robot with nonlinear friction using time-delay estimation, Ind. Electron. IEEE Trans. 55 (1) (2008) 258-269.

[26] G.R. Cho, P.H. Chang, S.H. Park, M. Jin, Robust tracking under nonlinear friction using time-delay control with internal model, Control Syst. Technol. IEEE Trans. 17 (6) (2009) 1406-1414.

[27] M. Jin, Y. Jin, P.H. Chang, C. Choi, High-accuracy trajectory tracking of industrial robot manipulators using time delay estimation and terminal sliding mode, in: Proceedings of the Thirty-fifth Annual Conference of IEEE Industrial Electronics, 2009. IECON'09, IEEE, 2009, pp. 3095-3099.

[28] P.H. Chang, J.W. Jeong, Enhanced operational space formulation for multiple tasks by using time-delay estimation, Robot. IEEE Trans. 28 (4) (2012) 773-786.

[29] D. Kim, R.B. Gillespie, P.H. Chang, Simple, robust control and synchronization of the lorenz system, Nonlinear Dyn. (2013) 1-10.

[30] J. Lee, P.H. Chang, R.S. Jamisola, Relative impedance control for dual-arm robots performing asymmetric bimanual tasks, IEEE Trans. Ind. Electron. 61 (7) (2013) 3786-3796.

[31] Y. Jin, P.H. Chang, M. Jin, D.G. Gweon, Stability guaranteed time-delay control of manipulators using nonlinear damping and terminal sliding mode, Ind. Electron. IEEE Trans. 60 (8) (2013) 3304-3317.

[32] D. Kim, K. Koh, G.R. Cho, L.-Q. Zhang, A robust impedance controller design for series elastic actuators using the singular perturbation theory, IEEE/ASME Trans. Mechatron. 25 (1) (2019) 164-174.

[33] J. Lee, H. Dallali, M. Jin, D.G. Caldwell, N.G. Tsagarakis, Robust and adaptive dynamic controller for fully-actuated robots in operational space under uncertainties, Autonom. Robot. 43 (4) (2019) 1023-1040.

[34] J. Lee, N. Deshpande, D.G. Caldwell, L.S. Mattos, Microscale precision control of a computer-assisted transoral laser microsurgery system, IEEE/ASME Trans. Mechatron. 25 (2) (2020) 604-615.

[35] J. Tuch, A. Feuer, Z.J. Palmor, Time delay estimation in continuous linear time-invariant systems, IEEE Trans. Autom. Control 39 (4) (1994) 823-827.

[36] D.K. Han, P.-H. Chang, Robust tracking of robot manipulator with nonlinear friction using time delay control with gradient estimator, J. Mech. Sci. Technol. 24 (8) (2010) 1743-1752.

[37] J.Y. Lee, M. Jin, P.H. Chang, Variable PID gain tuning method using backstepping control with time-delay estimation and nonlinear damping, IEEE Trans. Ind. Electron. 61 (12) (2014) 6975-6985.

[38] D. Fredlund, Density and compressibility characteristics of air-water mixtures, Canad. Geotech. J. 13 (4) (1976) 386-396.

[39] M.W. Spong, S. Hutchinson, M. Vidyasagar, Robot Modeling and Control, John Wiley \& Sons New York, First Edition.

[40] E. Fridman, A. Seuret, J.-P. Richard, Robust sampled-data stabilization of linear systems: an input delay approach, Automatica 40 (8) (2004) 1441-1446.

[41] H. Alazki, P. Ordaz, A. Poznyak, Robust bounded control for the flexible arm robot, in: Proceedings of the Fifty-second IEEE Conference on Decision and Control, IEEE, 2013, pp. 3061-3066.

[42] S.E. Talole, J.P. Kolhe, S.B. Phadke, Extended-state-observer-based control of flexible-joint system with experimental validation, IEEE Trans. Ind. Electron. 57 (4) (2010) 1411-1419.

[43] E. Fridman, U. Shaked, An improved stabilization method for linear time-delay systems, Autom. Control IEEE Trans. 47 (11) (2002) 1931-1937. 